\title{
QUASILINEARIZATION FOR SOME NONLOCAL PROBLEMS ${ }^{1}$
}

\author{
YUNFENG YIN \\ Florida Institute of Technology \\ Department of Applied Mathematics \\ Melbourne, FL 32901-6988 U.S.A.
}

\begin{abstract}
The method of generalized quasilinearization [4] is applied to study semilinear parabolic equation $u_{t}-L u=f(t, x, u)$ with nonlocal boundary conditions $u(t, x)=\int \phi(x, y) u(t, y) d y$ in this paper. The convexity of $f$ in $u$ is relaxed by requiring $f(t, x, u)+M u^{2}$ to be convex for some $M>0$. The quadratic convergence of monotone sequence is obtained.
\end{abstract}

Key words: Quasilinearization, nonlocal problem, semilinear parabolic equation, upper and lower solutions, monotone iterative technique, quadratic convergence.

AMS (MOS) subject classifications: $\quad 35 \mathrm{~K} 20,35 \mathrm{~K} 60,35 \mathrm{~K} 99$.

\section{INTRODUCTION}

In this paper, we consider the nonlocal boundary value problem (NBVP for short):

$$
\begin{gathered}
u_{t}-L u=f(t, x, u) \text { in } D_{T}=(0, T] \times \Omega, \\
u(0, x)=u_{0}(x) \quad \forall x \in \Omega \\
u(t, x)=\int_{\Omega} \phi(x, y) u(t, y) d y \quad \forall(t, x) \in \Gamma_{T},
\end{gathered}
$$

where $\Omega$ is a bounded domain in $R^{n}, \partial \Omega \in C^{2}, \Gamma_{T}=(0, T) \times \partial \Omega$,

$$
L=\sum_{i, j=1}^{n} a_{i j}(t, x) \frac{\partial^{2}}{\partial x^{2}}+\sum_{i=1}^{n} b_{i}(t, x) \frac{\partial}{\partial x}, \quad a_{i j}, b_{i} \in C^{0, \alpha}, 0<\alpha<1 .
$$

$u_{0}(x) \in C(\bar{\Omega}), f \in C\left[\bar{D}_{T} \times R, R\right]$ and satisfies Lipschitz condition in $u$. $\phi \in C[\partial \Omega \times \bar{\Omega}, R]$ satisfies $\phi(x, y) \geq 0, \int_{\Omega} \phi(x, y) d y \neq 0, \forall x \in \partial \Omega$ and $\int_{\Omega} \phi(x, y) d y \leq \rho<1, \forall x \in \partial \Omega$.

\footnotetext{
${ }^{1}$ Received: February, 1993. Revised: April, 1993.
} 
It is shown in [5] that, if $v, w$ are lower and upper solutions of NBVP such that $v \leq w$, then there exist monotone sequences which converge uniformly to the unique solution of NBVP. The method of upper and lower solutions and monotone iterative techniques played most important roles in constructing monotone sequences.

Consider the following ordinary differential equation with initial condition :

$$
u^{\prime}=g(t, u), u(0)=u_{0}, t \in J=[0, T]
$$

It is well known $[1,2]$ that the method of quasilinearization provides not only the monotone sequence but also quadratic convergence of the sequence for (1.4) if requiring more on $g(t, u)$, namely, $g(t, u)$ to be convex in $u$ for $t \in J$. The result is of computational interest. In [4], this method has been extended to more general cases, namely, without demanding the convexity of $g(t, u)$, by requiring $g(t, u)+M u^{2}$ to be convex for some $M>0$ and the same results have been obtained [4].

Our purpose, in this paper, is to apply the method used in [4] to study nonlocal problem (1.1) - (1.3)

\section{PRELIMINARIES}

Consider NBVP (1.1)-(1.3). A function $v_{0} \in C^{1,2}\left(D_{T}\right) \cap C\left(\bar{D}_{T}\right)$ is called a lower solution of NBVP if

$$
\begin{gathered}
\left(v_{0}\right)_{t}-L v_{0} \leq f\left(t, x, v_{0}\right) \quad \text { in } D_{T}, \\
v_{0}(0, x) \leq u_{0}(x) \quad \forall x \in \Omega, \\
v_{0}(t, x) \leq \int_{\Omega} \phi(x, y) v_{0}(t, y) d y \quad \text { on } \Gamma_{T} .
\end{gathered}
$$

An upper solution $w_{0}$ is defined analogously by reversing the above inequalities with respect to $w_{0}$. It is $\operatorname{shown}[5]$ that $v_{0} \leq w_{0}$ on $\bar{\Omega}$.

We first state two lemmas which we need in the proof of the main result. Define $Q_{T}=\left\{(t, x, u): v_{0} \leq u \leq w_{0},(t, x) \in \bar{D}_{T}\right\}$. If the existence of upper and lower solutions of NBVP is known, then we can prove the existence of solutions of NBVP in the closed set $Q_{T}$ which is the statement of Lemma 2.1. For proof, see [5].

Lemma 2.1: Consider

$$
u_{t}-L u=G(t, x, \eta(t, x), u(t, x)) \text { in } D_{T}
$$


with (1.2) and (1.3), where $\eta(t, x)$ is a given function on $\bar{D}_{T}$. Suppose that

(i) $v_{0}, w_{0}$ are lower solutions of (2.1), (1.2) and (1.3),

(ii) $G(t, x, \eta, u)$ satisfies Lipschitz condition in $u$, i.e., $\exists N>0$ such that

$$
-N\left(u_{1}-u_{2}\right) \leq G\left(t, x, \eta, u_{1}\right)-G\left(t, x, \eta, u_{2}\right) \leq N\left(u_{1}-u_{2}\right)
$$

whenever $u_{1} \geq u_{2}$, for $\left(t, x, u_{1}\right),\left(t, x, u_{2}\right) \in Q_{T}$. Then there exists a unique solution $u$ of $N B V P$ such that $u \in C^{1,2}\left(D_{T}\right) \cap C\left(\bar{D}_{T}\right)$ and $v_{0} \leq u \leq w_{0}$ on $\bar{D}_{T}$.

Lemma 2.2: Assume that $p \in C^{1,2}\left(D_{T}\right) \cap C\left(\bar{D}_{T}\right)$ satisfies

$$
\begin{gathered}
p_{t}(L p+K p+C) \leq 0 \quad \text { in } D_{T}, \\
p(0, x) \leq 0 \quad \forall x \in \Omega, \\
p(t, x) \leq \int_{\Omega} \phi(x, y) p(t, y) d y \quad \forall(t, x) \in \Gamma_{T},
\end{gathered}
$$

where $K>0, C>0$ are constants. Then, $p(t, x) \leq C e^{(K+1) t}$ on $\bar{D}_{T}$.

Proof: $\quad$ Let $\Psi=C e^{(K+1) t}$, then $\Psi$ verifies

$$
\begin{gathered}
\Psi_{t}-(L \Psi+K \Psi+C)=C\left(e^{(K+1) t}-1\right)>0 \text { in } D_{T} \\
\Psi(0, x)>0 \quad \forall x \in \Omega \\
\Psi(t, x)>\int_{\Omega} \phi(x, y) \Psi(t, y) d y \quad \text { on } \Gamma_{T} .
\end{gathered}
$$

By Theorem 2.1 of $[5], p(t, x) \leq \Psi$ on $\bar{D}_{T}$. This completes the proof of the lemma.

\section{MAIN RESULTS}

In this section, we shall apply the method of quasilinearization generalized in [3] to NBVP in order to obtain the monotone sequence and quadratic convergence of the sequence. Suppose now, $f \in C^{2}$ in $u$ and $f+M u^{2}$ is uniformly convex for $(t, x, u) \in Q_{T}$. Define $F(t, x, u) \equiv f(t, x, u)+M u^{2}$, then

$$
F_{u u}=f_{u u}+2 M>0 \text {. }
$$

If $u_{1} \geq u_{2}$, it follows

$$
\begin{gathered}
F_{u}\left(t, x, u_{1}\right)=f_{u}\left(t, x, u_{1}\right)+2 M u_{1} \geq F_{u}\left(t, x, u_{2}\right)+2 M u_{2} \\
F\left(t, x, u_{1}\right)-F_{u}\left(t, x, u_{2}\right) u_{1} \geq F\left(t, x, u_{2}\right)-F_{u}\left(t, x, u_{2}\right) u_{2} .
\end{gathered}
$$


We also assume that there exist constants $K_{1}>0, C_{1}>0$ such that

$$
\left|F_{u}\right| \leq K_{1} \text { and } F_{u u} \leq C_{1} \text {, for }(t, x, u) \in Q_{T} .
$$

Now, we are in the position to prove the main results.

\section{Theorem 3.1: $\quad$ Suppose that}

(i) $\quad v_{0}, w_{0}$ are lower and upper solutions of $N B V P$ such that $v_{0} \leq w_{0}$ on $\bar{D}_{T}$;

(ii) $F(t, x, u) \equiv f(t, x, u)+M u^{2}$ is uniformly convex in $Q_{T}$.

Then, there exists a monotone sequence $\left\{v_{n}\right\}$ which converges uniformly to the unique solution of NBVP and moreover, the convergence is quadratic.

Proof: $\quad$ Consider the modified nonlocal problem $\left(P^{*}\right)$ :

$$
\begin{gathered}
u_{t}-L u=f(t, x, \eta)+\left(f_{u}(t, x, \eta)+2 M \eta\right)(u-\eta)-M\left(u^{2}-\eta^{2}\right) \\
\equiv F(t, x, \eta)+F_{u}(t, x, \eta)(u-\eta)-M u^{2}
\end{gathered}
$$

with (1.2) and (1.3), where $\eta \in C^{1,2}\left(D_{T}\right) \cap\left(\bar{D}_{T}\right)$ is such that

$$
v_{0}(t, x) \leq \eta(t, x) \leq w_{0}(t, x) \text { on } D_{T}
$$

Define

$$
G(t, x, \eta, u) \equiv F(t, x, \eta)+F_{u}(t, x, \eta)(u-\eta)-M u^{2} .
$$

Since $v_{0}$ is a lower solution of NBVP and if $\eta=v_{0}$, because of (3.4), we have

$$
\left(v_{0}\right)_{t}-L v_{0} \leq f\left(t, x, v_{0}\right) \equiv G\left(t, x, v_{0}, v_{0}\right) \text { in } D_{T},
$$

From (3.3) and (3.4),

$$
u_{t}-L u=G\left(t, x, v_{0}, u\right) \text { in } D_{T}
$$

Similarly,

$$
\begin{gathered}
\left(w_{0}\right)_{t}-L w_{0} \geq f\left(t, x, w_{0}\right) \\
\geq F\left(t, x, v_{0}\right)+F_{u}\left(t, x, v_{0}\right)\left(w_{0}-v_{0}\right)-M w_{0}^{2} \equiv G\left(t, x, v_{0}, w_{0}\right) \text { in } D_{T} .
\end{gathered}
$$

From (3.5), (3.6) and (3.7) together with the initial and boundary conditions, we conclude that $v_{0}(t, x)$ and $w_{0}(t, x)$ are lower and upper solutions of $\left(P^{*}\right)$ with $\eta=v_{0}$. It is easy to see that $G\left(t, x, v_{0}, u\right)$ satisfies Lipschitz condition $(2.2)$ for $(t, x, u) \in Q_{T}$ since $F(t, x, u)$ is convex in $u$ and $v_{0}(t, x)$ is a given function. Therefore, by Lemma 2.1, there exists a unique solution $u$ of 
$\left(P^{*}\right)$ with $\eta=v_{0}$ such that $u \in C^{1,2}\left(D_{T}\right) \cap C\left(\bar{D}_{T}\right)$ and $v_{0} \leq u \leq w_{0}$ on $\bar{D}_{T}$.

Now, we construct the sequence $\left\{v_{n}\right\}$ by

$$
\begin{gathered}
\left(v_{n+1}\right)_{t}-L v_{n+1}=f\left(t, x, v_{n}\right)+\left(f_{u}\left(t, x, v_{n}\right)+2 M v_{n}\right)\left(v_{n+1}-v_{n}\right)-M\left(v_{n+1}^{2}-v_{n}^{2}\right) \\
\equiv F\left(t, x, v_{n}\right)+F_{u}\left(t, x, v_{n}\right)\left(v_{n+1}-v_{n}\right)-M v_{n+1}^{2} \text { in } D_{T}, \\
v_{n+1}(0, x)=u_{0}(x) \quad \forall x \in \Omega, \\
v_{n+1}(t, x)=\int_{\Omega} \phi(x, y) v_{n+1}(t, y) d y \text { on } \Gamma_{T},
\end{gathered}
$$

for $n=0,1,2, \ldots$.

Clearly, from the above discussion, we obtain that $\left(P^{*}\right)$ has a unique solution $v_{1}$ with $\eta=v_{0}$ and $v_{0} \leq v_{1} \leq w_{0}$ on $\bar{D}_{T}$.

Assume that for some $n,\left(P^{*}\right)$ has a unique solution $v_{n}$ with $\eta=v_{n-1}$ and $v_{n-1} \leq v_{n} \leq w_{0}$ on $\bar{D}_{T}$, by (3.2) and (3.8)

$$
\begin{aligned}
\left(v_{n}\right)_{t}-L v_{n} & =F\left(t, x, v_{n-1}\right)+F_{u}\left(t, x, v_{n-1}\right)\left(v_{n}-v_{n-1}\right)-M v_{n}^{2} \\
& \leq F\left(t, x, v_{n}\right)-M v_{n}^{2}=f\left(t, x, v_{n}\right) \text { in } D_{T} .
\end{aligned}
$$

On the other hand,

$$
\begin{gathered}
\left(v_{n+1}\right)_{t}-L v_{n+1}=F\left(t, x, v_{n}\right)+F_{u}\left(t, x, v_{n}\right)\left(v_{n+1}-v_{n}\right)-M v_{n+1}^{2} \\
=G\left(t, x, v_{n}, v_{n+1}\right) \text { in } D_{T} .
\end{gathered}
$$

Now, (3.9) becomes

$$
\left(v_{n}\right)_{t}-L v_{n} \leq f\left(t, x, v_{n}\right) \equiv G\left(t, x, v_{n}, v_{n}\right) \text { in } D_{T}
$$

Similarly,

$$
\left(w_{0}\right)_{t}-L w_{0} \geq f\left(t, x, w_{0}\right) \equiv G\left(t, x, v_{n}, w_{0}\right) \text { in } D_{T}
$$

Applying the same arguments as above, we claim that (3.10), (3.11) and (3.12) together with the initial and boundary conditions yield the unique solution $v_{n+1}$ of $\left(P^{*}\right)$ with $\eta=v_{n}$ and $v_{n} \leq v_{n+1} \leq w_{0}$ on $\bar{D}_{T}$. Thus, by induction, we conclude that for all $n$,

$$
v_{n} \leq v_{1} \leq \cdots \leq v_{n} \leq \cdots \text { on } \bar{D}_{T}
$$

and $\left\{v_{n}\right\}$ is bounded above by $w_{0}$ on $\bar{D}_{T}$. Therefore, we obtain a sequence $\left\{v_{n}\right\}$ which is monotone increasing and uniformly bounded in $C^{1,2}\left(D_{T}\right) \cap C\left(\bar{D}_{T}\right)$. By standard arguments $[3,5],\left\{v_{n}\right\}$ converges uniformly to the unique solution of NBVP. 
It remains to show that convergence of $\left\{v_{n}\right\}$ to the solution $u$ of NBVP is quadratic. Observe that, for all $n \geq 0, \quad v_{n} \leq u$. Set $p_{n}=u(t, x)-v_{n}(t, x)$ on $\bar{D}_{T}$. Define $\left\|p_{n}\right\| \underset{(t, x) \in \bar{D}_{T}}{=\sup }\left|u(t, x)-v_{n}(t, x)\right|$. Then,

$$
\begin{gathered}
\left(p_{n}\right)_{t}-L p_{n}=f(t, x, u)-\left[F\left(t, x, v_{n-1}\right)+F_{u}\left(t, x, v_{n-1}\right)\left(v_{n}-v_{n-1}\right)-M v_{n}^{2}\right] \\
=F_{u}(t, x, \theta)\left(u-v_{n-1}\right)-F_{u}\left(t, x, v_{n-1}\right)\left(u-v_{n-1}\right)+F_{u}\left(t, x, v_{n-1}\right)\left(u-v_{n}\right)+M\left(v_{n}^{2}-u^{2}\right) \\
=F_{u u}(t, x, \xi)\left(u-v_{n-1}\right)\left(\theta-v_{n-1}\right)+F_{u}\left(t, x, v_{n-1}\right)\left(u-v_{n}\right)+M\left(v_{n}^{2}-u^{2}\right) \text { in } D_{T},
\end{gathered}
$$

where $v_{n-1} \leq \xi \leq \theta \leq u$ for $(t, x) \in D_{T}$.

Since $0<F_{u u} \leq C_{1},\left|F_{u}\right| \leq K_{1}$ on $Q_{T}$ and $v_{0} \leq v_{n}, u \leq w_{0}$, we have

$$
\begin{aligned}
& \left(p_{n}\right)_{t}-L p_{n} \leq C_{1}\left(u-v_{n-1}\right)^{2}+K p_{n}-M\left(v_{n}+u\right) p_{n} \\
& \leq\left(K_{1}-2 M v_{0}\right) p_{n}+C_{1}\left\|p_{n-1}\right\|^{2} \leq K p_{n}+C \text { in } D_{T}
\end{aligned}
$$

where $K=K_{1}+2 M V$ and $\left|v_{0}\right| \leq V$ on $\bar{D}_{T}, C=C_{1}\left\|p_{n-1}\right\|^{2}$. Therefore, by Lemma 2.2, we get

$$
p_{n}(t, x) \leq C e^{(K+1) t} \leq C_{1} e^{(K+1) T}\left\|p_{n-1}\right\|^{2}=C_{1} e_{(t, x) \in \bar{D}_{T}}^{(K+1) T} \text { sup }\left|u-v_{n-1}\right|^{2} \text { on } \bar{D}_{T}
$$

which yields

$$
\sup _{(t, x) \in \bar{D}_{T}}\left|u-v_{n}\right| \leq \underset{(t, x) \in \bar{D}_{T}}{\alpha s u p}\left|u-v_{n-1}\right|^{2}, \alpha=C_{1} e^{(K+1) T}
$$

The proof of Theorem 3.1 is therefore complete.

\section{REFERENCES}

[1] Bellman, R., Methods of Nonlinear Analysis, Vol. II, Academic Press, New York 1973.

[2] Bellman, R., and R. Kalaba, Quasilinearization and Nonlinear Boundary Value Problems, American Elsevier, New York 1965.

[3] Ladde, G.S., Lakshmikantham, V., and S. Leela, Monotone Iterative Techniques for Nonlinear Differential Equations, Pitman, Boston 1985.

[4] Lakshmikantham, V., and S. Malek, Generalized Quasilinearization, preprint.

[5] Yin, Y., On nonlinear parabolic equations with nonlocal boundary condition, preprint. 


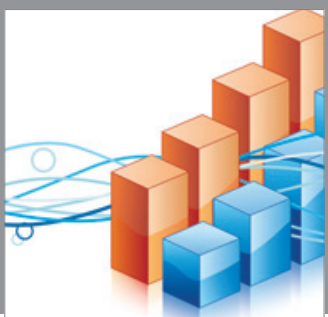

Advances in

Operations Research

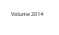

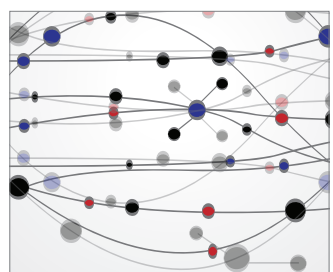

\section{The Scientific} World Journal
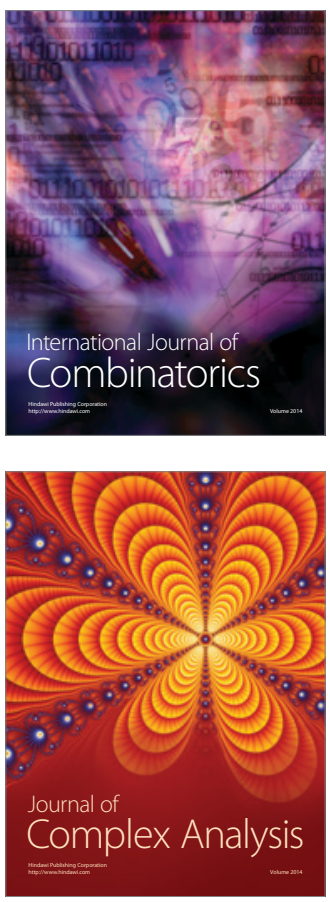

International Journal of

Mathematics and

Mathematical

Sciences
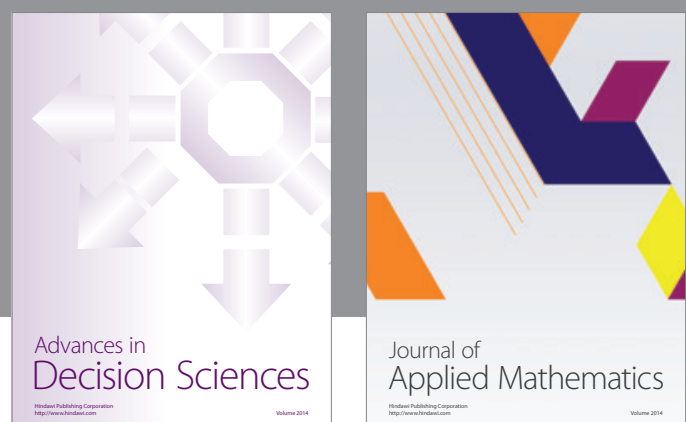

Journal of

Applied Mathematics
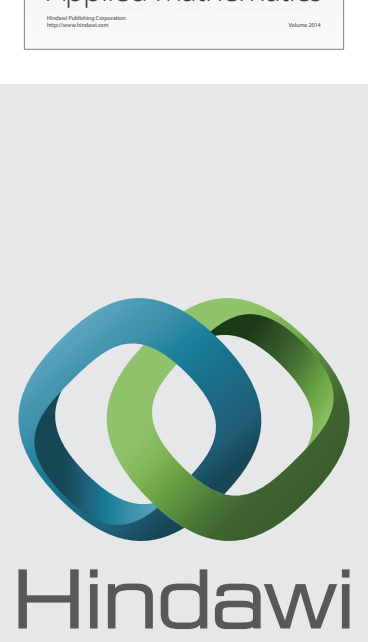

Submit your manuscripts at http://www.hindawi.com
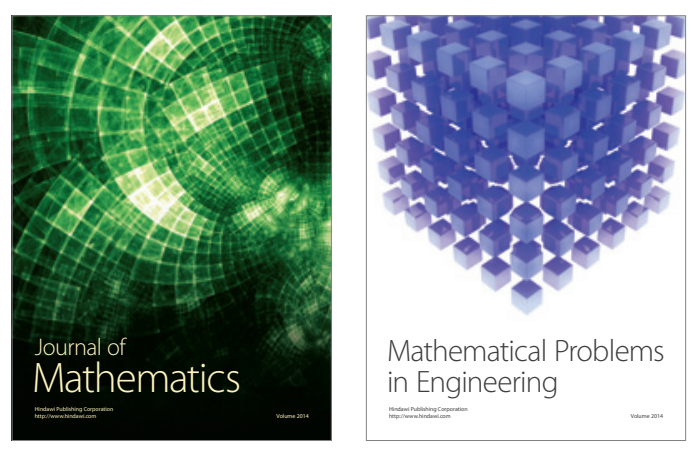

Mathematical Problems in Engineering
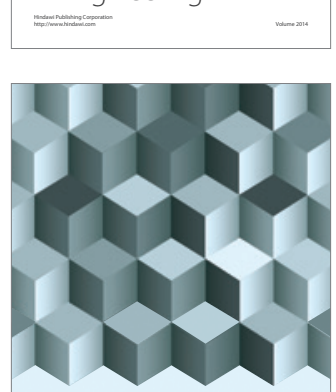

Journal of

Function Spaces
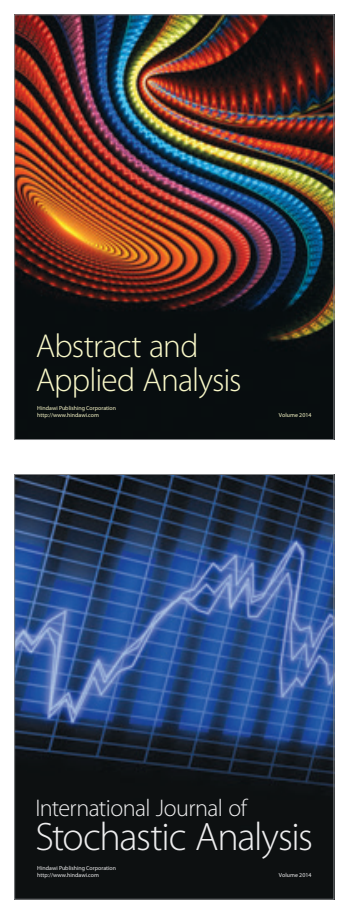

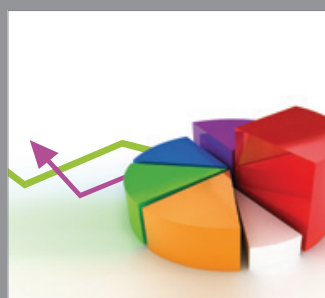

ournal of

Probability and Statistics

Promensencen
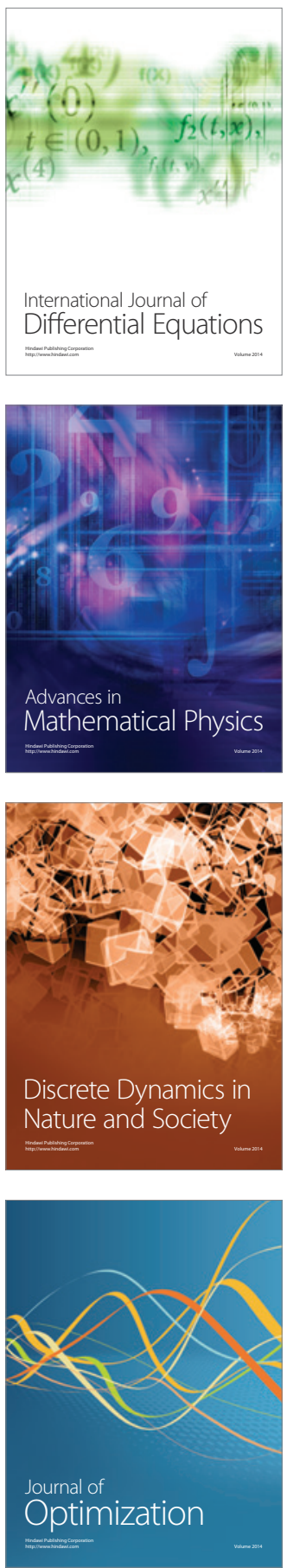